\title{
AVALIAÇÃO DA APLICAÇÃO DOS FILTROS DE IMAGEM NA DETECÇÃO DE FRATURAS RADICULARES
}

\section{EVALUATION OF ENHANCEMENT FILTERS IN THE DETECTION OF VERTICAL ROOT FRACTURES}

\author{
Anna Carolina de OLIVEIRA'; Marcus Vinícius Corrêa da COSTA²; Fernanda Ferreira NUNES'; Jeane Katiuscia SILVA3; Cristiane \\ Martins Rodrigues BERNARDES4; Carlos ESTRELA²; Brunno Santos de Freitas SILVA5; Fernanda Paula YAMAMOTO-SILVA ${ }^{2}$ \\ 1 - Graduanda em odontologia. Departamento de Ciências Estomatológicas, Faculdade de Odontologia, Universidade de Goiás - UFG. \\ 2 - Doutor(a). Departamento de Ciências Estomatológicas, Faculdade de Odontologia, Universidade de Goiás - UFG. \\ 3 - Graduanda em odontologia. Departamento de Diagnóstico Oral, Curso de Odontologia, Centro Universitário de Anápolis - UniEVANGÉLICA. \\ 4 - Mestre. Departamento de Diagnóstico Oral, Curso de Odontologia, Centro Universitário de Anápolis - UniEVANGÉLICA. \\ 5 - Doutor. Departamento de Diagnóstico Oral, Curso de Odontologia, Centro Universitário de Anápolis - UniEVANGÉLICA.
}

\section{RESUMO}

Objetivo: Avaliar a acurácia, sensibilidade e especificidade de diferentes filtros do software Cliniview ${ }^{\mathrm{TM}}$ (versão 10.1) em radiografias periapicais digitais na detecção de fraturas radiculares verticais em dentes unirradiculares. Material e Métodos: Foram utilizados 31 dentes humanos recém-extraídos divididos em dois grupos: controle (CTL) e fraturado (FTR), constituídos por 15 e 16 dentes, respectivamente. Realizou-se o tratamento endodôntico dos dentes para posterior desobturação de $2 / 3$ da raiz e confecção dos pinos metálicos. Após cimentação dos pinos, as raízes dos dentes grupo FTR foram fraturadas na máquina de ensaio (Instron). As radiografias periapicais digitais foram obtidas em todos os dentes com sistema digital Express ${ }^{\mathrm{TM}}$. As radiografias originais e com aplicação dos filtros (inversão na escala de cinza, hot, nitidez 1 , vertical e vertical + horizontal) foram salvas e avaliadas por dois especialistas. Após as análises, foram calculados os valores de sensibilidade, especificidade, preditivo positivo, preditivo negativo e acurácia da radiografia periapical digital com e sem a aplicação dos filtros. Resultados: Considerando os dois examinadores, os maiores valores de acuraria, sensibilidade e especificidade para as imagens originais foram de $85,2 \%, 100 \%$ e $95,2 \%$ respectivamente. Para as radiografias com aplicação de filtro, os maiores valores de acurácia, sensibilidade e especificidade foram de $82,6 \%, 71,4 \%$ e $87,5 \%$, respectivamente. Conclusão: O uso de filtros não aumentou os valores de acurácia, sensibilidade e especificidade das radiografias periapicais digitais para a detecção das fraturas radiculares verticais. De fato, pelo contrário, em alguns casos, o diagnóstico piorou.

Palavras-chaves: Radiografia dentária; Fraturas dos dentes; Diagnóstico.

\section{INTRODUÇÃO}

A fratura radicular vertical (FRV) é a lesão dentária com o pior prognóstico na endodontia ${ }^{1}$. São definidas como fraturas longitudinais que acontecem na raiz dos dentes, acometendo ou não a coroa, e são caracterizadas por iniciarem no ápice radicular e continuar coronalmente, podendo envolver o ligamento periodontal. A separação dos fragmentos dentários aumenta com o tempo, e no osso adjacente inicia-se o processo de reabsorção e destruição periodontal, que ampliam gradativamente com o tempo, afetando negativamente o prognóstico do dente acometido ${ }^{2}$.

A etiologia da FRV é comumente iatrogênica, sendo decorrente da inserção de núcleos/pinos de retenção em dentes não vitais ou da força excessiva durante a condensação lateral do material obturador endodôntico ${ }^{3}$.

É um desafio para a radiologia e endodontia a detecção de FRV em seu início. Na maioria dos casos, o diagnóstico só é alcançado após a combinação dos sinais clínicos e radiográficos. Clinicamente, percebe-se dor, edema local, mobilidade dentária, bolsa periodontal, sinusite, abcesso, sensibilidade à palpação ou percussão ${ }^{4}$.
A radiografia periapical é usualmente o primeiro exame de imagem utilizado para avaliar a fratura radicular ${ }^{5}$, devido a sua alta resolução espacial, baixo custo e simplicidade técnica. Porém, a radiografia periapical permite apenas uma visão bidimensional das estruturas anatômicas, além de apresentar a sobreposição das estruturas mineralizadas que se antepõem entre a fonte de raios- $X$ e o receptor digital ${ }^{6}$. Ainda assim, para a fratura ser detectada, o feixe central de raios- $x$ deve estar no plano da fratura ou em uma variação de $4^{\circ}$ para ambos os lados $^{3}$.

Apesar de apresentar limitações, a radiografia periapical digital permite o ajuste de contraste e densidade, além da aplicação de filtros que visam melhorar a qualidade da imagem através de softwares no pós-processamento. Poucos estudos têm avaliado a aplicação dos filtros para detecção de fraturas radiculares ${ }^{5,7-9}$. Segundo Angelopoulos et al. ${ }^{10}$ (2004), esses ajustes podem não ser suficientes para a detecção de doenças e anormalidades dentárias. Dessa forma, julga-se lícita a investigação dos filtros na detecção de FRV. 
Assim, o objetivo do presente estudo foi avaliar a acurácia, a sensibilidade e a especificidade da radiografia periapical com a aplicação de filtros na detecção de fraturas radiculares verticais.

\section{MATERIAL E MÉTODOS}

Este estudo foi aprovado pelo Comitê de Ética em pesquisa da Universidade Federal de Goiás sob o número 477.315, no ano de 2014.

\section{Obtenção da amostra e armazenamento dos dentes}

Foram utilizados 31 dentes humanos unirradiculares, recémextraídos, de pacientes das Clínicas da Faculdade de Odontologia da Universidade Federal de Goiás, sendo incluídos na pesquisa apenas dentes doados por pacientes mediante assinatura do Termo de Consentimento Livre e Esclarecido. Estes dentes foram condicionados em solução de timol a $0,2 \%$ e em seguida imersos em solução de hipoclorito a 5\% (Fitofarma, Goiânia, GO, Brasil) por 30 minutos para remoção de tecido orgânico na superfície externa das raízes. Posteriormente os dentes foram raspados com cureta periodontal para o término da remoção de restos de ligamento periodontal e tecido orgânico.

\section{Tratamento endodôntico e colocação do retentor metálico}

Os dentes foram tratados endodonticamente com instrumentos rotatórios a partir do sistema Pro Taper (Dentsplay Maillefer, Tulsa, UK) e obturados com gutta percha cone único (Dentsplay Maillefer, Tulsa, UK). Após esse tratamento, desobturou-se 2/3 do canal radicular para possibilitar a colocação de um retentor metálico em cada dente, que foi cimentado com fosfato de zinco, seguindo as instruções do fabricante.

\section{Exames radiográficos}

Para a aquisição da imagem radiográfica, os dentes foram colocados em alvéolos de mandíbula seca. Para cada amostra (dente) de ambos os grupos, foram realizadas radiografias periapicais digitais utilizando o aparelho FOCUS ${ }^{\mathrm{TM}}$ (Kavo do Brasil Ind. Com. Ltda.), pela técnica do paralelismo utilizandose incidência ortocêntrica. $O$ receptor de imagem utilizado foi a placa de fósforo tamanho $\mathrm{n}^{\mathrm{o}} 2-3 \times 4 \mathrm{~cm}$, do sistema de imagens Intraoral Digital Express ${ }^{\mathrm{TM}}$ (Instrumentarium Dental, Tuusula, Finland) que, em seguida, foi escaneada no aparelho Express ${ }^{\mathrm{TM}}$, armazenada e manipulada no programa de computador CliniView $w^{\mathrm{TM}}$ que acompanha o sistema digital.

\section{Confecção das fraturas radiculares verticais}

Foram criados dois grupos: Grupo controle (CTL) e Grupo Fraturado (FTR), sendo ambos constituídos com 15 e 16 dentes, respectivamente, randomicamente numerados. As fraturas foram realizadas somente no Grupo Fraturado (FTR).

Para confecção das fraturas simulou-se o ligamento periodontal, incluindo os dentes em resina de poliestireno com o fluido do silicone de adição Futura AD (Nova DFL). Primeiramente, as raízes foram rapidamente mergulhadas em cera n. 7 liquefeita de modo que ficassem recobertas com uma camada de cera de aproximadamente 0,2 a $0,3 \mathrm{~mm}$, e a espessura aferida com paquímetro. Em películas de filme radiográfico demarcou-se centralizadamente uma circunferência referente ao diâmetro $(25 \mathrm{~mm})$ de um cilindro de PVC, e no centro desta circunferência, um furo compatível com o diâmetro das raízes. Em seguida, a raiz foi inserida na perfuração até a marca de $2 \mathrm{~mm}$ referente às distâncias biológicas, de forma que seu longo-eixo ficasse perpendicular à superfície da película radiográfica. Neste momento, estabilizou-se a raiz com cera pegajosa. $\mathrm{O}$ cilindro de PVC foi posicionado e fixado com cera em torno da raiz do dente vaselinado. Este conjunto foi posicionado de forma invertida com a raiz voltada para cima em uma placa com perfurações circulares de $15 \mathrm{~mm}$. Resina de poliestireno autopolimerizável foi manipulada conforme recomendações do fabricante e vertida no cilindro. Após a polimerização da resina, 2 horas depois da inclusão, removeu-se o conjunto da placa de suporte.

A reprodução artificial do ligamento periodontal foi realizada no momento do teste de resistência à fratura. Para esta etapa, os dentes foram removidos dos alvéolos artificiais e limpos, tanto raízes quanto alvéolos, retirando-se completamente a camada de cera. $\mathrm{O}$ material de moldagem fluido do silicone de adição foi manipulado seguindo-se as recomendações do fabricante, inserido no alvéolo e o dente introduzido sob pressão digital até a marcação de $2 \mathrm{~mm}$ do limite coronário da raiz. Após a polimerização, os excessos foram retirados com lâmina de bisturi.

Para que as fraturas fossem padronizadas, utilizou-se a máquina de Ensaio Universal - Instron, utilizando uma célula de carga $2000 \mathrm{kgf}$ e velocidade de 0,5mm/min. Após a fratura, os dentes foram colados com cianoacrilato e radiografados novamente.

Após a aquisição das radiografias digitais, as imagens originais foram salvas para posteriormente serem ajustadas no software Cliniview $^{\mathrm{TM}}$. Cinco possibilidades de ajustes para melhorar a qualidade da imagem foram realizadas para comparação: aplicação dos filtros Nitidez 1, Vertical, Horizontal+Vertical, Hot e Inversão na escala de cinza, separadamente (Figura 1).

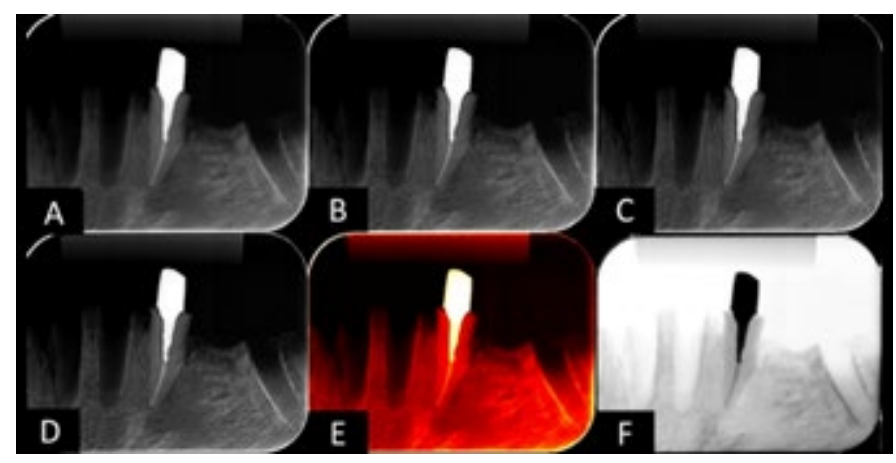

Figura 1 - Radiografias periapicais digitais. A- Radiografia original; B- Radiografia com filtro Nitidez 1; C- Radiografia com filtro Vertical; D- Radiografia com filtro Vertical+ Horizontal; E- Radiografia com filtro Hot; F- Radiografia com filtro Inversão na escala de cinza.

\section{Avaliação dos especialistas}

Participaram da análise 2 (dois) endodontistas com mais de 10 anos de experiência. As imagens foram disponibilizadas em arquivos de Power Point, uma com as radiografias originais, e uma com as radiografias com os filtros: Nitidez 1, Vertical, Horizontal+Vertical, Hot e Inversão na escala de cinza. As imagens foram avaliadas e através de um questionário estruturado, com perguntas sobre a presença ou ausência da fratura, classificando os dentes em: fraturado, incerteza e não fraturado. Posteriormente foram avaliadas as imagens com os 
filtros, e o especialista deveria classificar a fratura novamente, e posteriormente, dizer se o filtro melhorou, manteve, ou piorou a qualidade da imagem para a avaliação da fratura.

\section{Análise estatística}

A partir do software SPSS, avaliou-se a acurácia, a sensibilidade e a especificidade da radiografia periapical digital com ou sem filtro, e também a diferença entre os filtros, utilizando-se o teste qui-quadrado. Obteve-se ainda curva ROC.

\section{RESULTADOS}

Após avaliação dos especialistas, os dados foram tabulados e a acurácia, a sensibilidade e a especificidade, preditivo positivo e negativo da radiografia periapical com e sem a utilização dos filtros foram calculadas para cada avaliador (Tabela 1). A avaliação da performance dos filtros está representada na Tabela 2.

\section{DISCUSSÃO}

Os resultados desse trabalho mostram que não houve diferença significativa nos valores de acurácia, sensibilidade e especificidade da radiografia periapical digital com e sem a aplicação dos filtros, no diagnóstico de fraturas radiculares. Adicionalmente, os filtros hot, nitidez, vertical+horizontal e horizontal em alguns casos, pioraram o diagnóstico. Neste estudo, observou-se ainda, que em muitos casos, os avaliadores não conseguiram se decidir quanto a presença ou ausência de fratura.

Os filtros são ferramentas que tem como objetivo utilizar equações matemáticas no processamento de imagem ${ }^{11}$. Essas ferramentas podem melhorar a imagem original compensando a perda de qualidade causada por ruído ou subexposição de raios- $x^{12}$.

Alguns trabalhos têm avaliado os filtros na detecção de fraturas radiculares. Em 2005, Wenzel et al. ${ }^{5}$ não observaram diferença entre as imagens originais e com filtros no diagnóstico de fraturas radiculares horizontais. Neste estudo, os autores utilizaram dois sistemas distintos de imagem digital. Com o sistema Digora Optime $^{\mathrm{TM}}$, semelhante ao sistema utilizado no presente trabalho, os autores não observaram diferença significativa na utilização dos filtros. Curiosamente, quando compararam a acurácia entre os sistemas de aquisição de imagem radiográfica digital Digora Optime $^{\mathrm{TM}}$, e o sistema de dispositivo de carga acoplada CCD RVG-ui, os autores observaram melhora na acurácia, supondo que o tipo de sistema pode influenciar na qualidade da imagem de forma mais significativa que a aplicação dos filtros, já que os sistemas estudados apresentam resolução espacial diferente.

Ao contrário de Wenzel et al. ${ }^{5}$ (2005) e Tofangchiha et al. ${ }^{8}$ (2012), no presente estudo não se observou influência na utilização dos filtros no diagnóstico de fratura radicular, assim como Kamburolu et al. ${ }^{7}$ (2010), mesmo utilizando o sistema de dispositivo de carga acoplada CCD.

Nascimento et al. ${ }^{9}$ (2015) demonstrou que o filtro "sharpen" apresentou melhor performance quando comparado aos filtros: 3D, inversão na escala de cinza ou negativo e sombra no diagnóstico de fraturas radiculares in vitro, utilizando o sistema Digora Optime ${ }^{\mathrm{TM}}$. No presente estudo, o filtro "sharpen" melhorou o diagnóstico em $10 \%$ dos casos, porém, piorou em $31,6 \%$. O único filtro que não influenciou negativamente a detecção da
Tabela 1 - Sensibilidade, especificidade, preditivo positivo, negativo e acurácia do avaliador 1 e 2 com e sem filtro.

\begin{tabular}{llllll}
\hline & Sensibilidade & Especificidade & $\begin{array}{l}\text { Preditivo } \\
\text { Positivo }\end{array}$ & $\begin{array}{l}\text { Preditivo } \\
\text { Negativo }\end{array}$ & Acurácia \\
\hline $\begin{array}{l}\text { Avaliador } 1 \\
\text { Original }\end{array}$ & 1,000 & 0,810 & 0,600 & 1,000 & 0,852 \\
\hline Com Filtros & 0,714 & 0,875 & 0,714 & 0,875 & 0,826 \\
\hline $\begin{array}{l}\text { Avaliador } 2 \\
\text { Original }\end{array}$ & 0,500 & 0,952 & 0,800 & 0,833 & 0,828 \\
\hline Com Filtros & 0,625 & 0,800 & 0,625 & 0,800 & 0,739 \\
\hline
\end{tabular}

Tabela 2 - Avaliação da performance dos diferentes filtros em comparação a imagem radiográfica original.

\begin{tabular}{lllll}
\hline Filtros & Piorou & Manteve & Melhorou & $\mathrm{p}$ \\
\hline Inversão de escala de cinza & $0(0,0 \%)$ & $7(87,5 \%)$ & $1(12,5 \%)$ & 0,356 \\
\hline Hot & $2(16,7 \%)$ & $8(66,7 \%)$ & $2(16,7 \%)$ & 0,910 \\
\hline Nitidez & $6(31,6 \%)$ & $11(57,9 \%)$ & $2(10,5 \%)$ & 0,166 \\
\hline Vertical + Horizontal & $5(27,8 \%)$ & $12(66,7 \%)$ & $1(5,6 \%)$ & 0,283 \\
\hline Horizontal & $1(14,3 \%)$ & $6(85,7 \%)$ & $0(0,0 \%)$ & 0,503 \\
\hline
\end{tabular}

FRV foi o "inversão da escala de cinza", o qual inverte a escala de cinza, transformando as imagens radiolúcidas em radiopacas e vice-versa.

Tofangchiha et al. ${ }^{8}$ (2012), avaliaram dois filtros que também foram utilizados no presente estudo: inversão da escala de cinza e colorização, equivalente ao "Hot". Os autores concluíram que as imagens originais apresentaram maior acurácia quando comparado aos dois filtros, e que essa ferramenta não contribui para o diagnóstico de fratura. No estudo de Kamburolu et al. ${ }^{7}$ (2010), os autores também não detectaram diferença quando comparado o filtro "inversão da escala de cinza" com os filtros "sharpness", "zoom-in", e "pseudo-3D". A comparação com os trabalhos supracitados é dificultada, pois estes não utilizaram o mesmo software (CliniView $\left.{ }^{\mathrm{TM}}\right)$, que apesar de apresentar semelhanças técnicas com o Digora Optime ${ }^{\mathrm{TM}}$, pode apresentar diferença na produção dos algoritmos utilizados nos filtros.

Neste trabalho utilizou-se somente os filtros do software CliniView $w^{\mathrm{TM}}$. Segundo o fabricante, a função "Inversão da escala de cinza" inverte áreas brancas em áreas pretas, e a escala de cinza também é invertida. Os filtros "horizontal" e "vertical mais horizontal" realçam as bordas horizontal e vertical simultaneamente. Esperava-se, portanto, que realçando as linhas de fraturas, as mesmas fossem ser mais facilmente observadas. No entanto, apresentaram piora no diagnóstico em $14,3 \%$ e $27,8 \%$ respectivamente.

O filtro "hot" inverte a escala de cinza em uma determinada seleção de cores, uma vez que o olho humano é mais sensível para detectar diferenças nas cores do que em tons de cinza, melhorando, teoricamente, a qualidade da informação obtida na imagem radiográfica ${ }^{13}$, no entanto, apesar de melhorar a detecção da fratura em $16,7 \%$ dos casos, piorou o diagnóstico na mesma proporção.

No presente trabalho evidenciou-se que os avaliadores apresentaram alto índice de incerteza quanto ao diagnóstico de fratura, o que é um reflexo da dificuldade de se diagnosticar 
fraturas radiculares em radiografias periapicais. Outro fator limitante do trabalho in vitro é que diferentemente do paciente que apresenta fraturas radiculares, não se observa outros sinais radiográficos como aumento do espaço do ligamento periodontal e/ou rarefação óssea periapical, além da ausência de informações clínicas importantes, com dor ou sensibilidade.

\section{CONCLUSÃO}

A utilização dos filtros não contribuiu de forma significativa a acurácia, sensibilidade e especificidade das radiografias periapicais digitais na detecção de FRV mostrando ainda, piora no diagnóstico em alguns casos.

\section{REFERÊNCIAS}

01. Orhan K, Aksoy U, Kalender A. Cone-beam computed tomographic evaluation of spontaneously healed root fracture. J Endod. 2010; 36(9): 1584-7.

02. Rivera E, Walton RE. Cracking the cracked tooth code: Detection and treatment of various longitudinal tooth fractures. Am Assoc Endodontists Colleagues for Excellence News Lett. 2008; 2:1-8.

03. Youssefzadeh S, Gahleitner A, Dorffner R, Bernhart T, Kainberger FM. Dental Vertical Root Fractures: Value of CT in Detection. Radiology. 2013; 210(2): 545-9.

04. Bernardes RA, Moraes IG, Húngaro Duarte MA, Azevedo BC, Azevedo JR, Bramante CM. Use of cone-beam volumetric tomography in the diagnosis of root fractures. Oral Surgery, Oral Med Oral Pathol Oral Radiol Endodontology. 2009; 108(2): 270-7.

05. Wenzel A. A review of dentists' use of digital radiography and caries diagnosis with digital systems. Dentomaxillofacial Radiol. 2006; 35(5): 307-14.

06. Patel S, Dawood A, Whaites E, Pitt Ford T. New dimensions in endodontic imaging: Part 1. Conventional and alternative radiographic systems. Int Endod J. 2009; 42(6): 447-62.

07. Kamburòlu K, Murat S, Pehlivan SY. The effects of digital image enhancement on the detection of vertical root fracture. Dent Traumatol. 2010; 26(1): 47-51.

08. Tofangchiha M, Bakhshi M, Shariati M, Valizadeh S, Adel M, Sobouti F. Detection of vertical root fractures using digitally enhanced images: Reverse-contrast and colorization. Dent Traumatol. 2012; 28(6): 478-82.

09. Nascimento HAR, Ramos ACA, Neves FS, Azevedo-Vaz SL, Freitas DQ. The "Sharpen" filter improves the radiographic detection of vertical root fractures. Int Endod J. 2015; 48(5): 428-34.

10. Angelopoulos C, Bedard A, Katz JO, Karamanis S, Parissis N. Digital panoramic radiography: An overview. Semin Orthod. 2004; 10(3): 194-203.

11. Lehmann TM, Troeltsch E, Spitzer K. Image processing and enhancement provided by commercial dental software programs. Dentomaxillofacial Radiol. 2002; 31(4): 264-72.

12. Haiter-Neto F, Spinelli Casanova M, Frydenberg M, Wenzel A. Taskspecific enhancement filters in storage phosphor images from the Vistascan system for detection of proximal caries lesions of known size. Oral Surgery, Oral Med Oral Pathol Oral Radiol Endodontology. 2009; 107(1): 116-21.

13. Kats L, Vered M. Pseudo-color filter in two-dimensional imaging in dentistry. Refuat Hapeh Vehashinayim (1993). 2014; 31(4): 13-5.

\section{ABSTRACT}

Objective: To evaluate the accuracy, sensitivity, and specificity of different Cliniview ${ }^{\mathrm{TM}}$ (version 10.1) software filters in digital periapical radiographs for the detection of vertical root fractures in single-rooted teeth. Materials and Methods: Thirty-one human teeth freshly extracted were divided into two groups: control (CTL) and fractured (FTR), constituted by 15 and 16 teeth respectively. The endodontic treatment of the teeth was performed and followed by desobturation of $2 / 3$ of the root canal and the metallic posts confection. After the cementation of the posts, the fractures were made using the universal test machine (Instron) only in the FTR group. The digital periapical radiographs were performed in all the teeth using the Express ${ }^{\mathrm{TM}}$ digital system. The original radiographs and the ones with applied filters (gray scale, hot, sharpness 1, vertical and vertical
+ horizontal) were saved and evaluated by two specialists. After the analysis, the sensitivity, specificity, positive predictive value, negative predictive value and accuracy of the digital periapical radiographs with and without the application of filters were calculated. Results: Taking into consideration the two examiners, the highest accuracy, sensitivity and specificity values for the original images were $85,2 \%, 100 \%$ and $95,2 \%$ respectively. For the radiographs with applied filters, the highest accuracy, sensitivity and specificity values found were of $82,6 \%, 71,4 \%$ and $87,5 \%$, respectively. Conclusion: The use of filters did not increase the accuracy, sensitivity and specificity values of the digital periapical radiographs for the detection of VRF. In fact, on the contrary, for some cases, the diagnosis became worse.

Keywords: Dental radiography; Tooth fractures; Diagnosis.

\section{AUTOR PARA CORRESPONDÊNCIA}

Profa. Dra. Fernanda Paula Yamamoto-Silva

Universidade Federal de Goiás, Departamento de Ciências

Estomatológicas

Praça Universitária, s/n, Setor Universitário

CEP.: 74605-220, Goiânia, GO, Brasil.

E-mail: fernanda.paula.yamamoto@gmail.com

Telefone: 62 3434-4394 